\title{
Agenda 2030 en Argentina: percepciones de expertos en salud, comparación con expertos de Brasil y primeros impactos de la pandemia COVID-19
}

\author{
Agenda 2030 in Argentina: perceptions of health experts, comparison \\ with the perceptions of Brazilian experts and the first impacts of the \\ COVID-19 Pandemic
}

Mariano Fontela (https://orcid.org/0000-0003-3449-5809) ${ }^{1}$

Soledad Beltrame (https://orcid.org/0000-0001-7894-4261) ${ }^{2}$

Maurício Monsalvo (https://orcid.org/0000-0002-2037-2106) ${ }^{1}$

Érica Kastrup Bittencourt e Câmara (https://orcid.org/0000-0002-2953-9259) ${ }^{3}$

José Mendes Ribeiro (https://orcid.org/0000-0003-0182-395X) ${ }^{4}$

Marcelo Rasga Moreira (https://orcid.org/0000-0003-3356-7153) ${ }^{4}$

${ }^{1}$ Universidad Isalud. C1095AAQ, Venezuela 931.

C1095AAQ Buenos Aires Argentina.marianofontela@ hotmail.com

${ }^{2}$ Universidad Isalud. Buenos

Aires Argentina.

${ }^{3}$ Centro de Estudos

Estratégicos, Fundação Oswaldo Cruz. Rio de

Janeiro RJ Brasil.

${ }^{4}$ Departamento de Ciências

Sociais, Escola Nacional

de Saúde Pública Sergio

Arouca, Fundação Oswaldo

Cruz. Rio de Janeiro RJ

Brasil.

\begin{abstract}
The 2030 Agenda - a strategy of the United Nations Organization (UN) to promote global and sustainable human development capable of satisfying basic social needs - is still in the initial stages in most of the countries of South America. The scope of this investigation was to consult a group of health experts on the possibilities of Argentina fulfilling the 2030 Agenda, especially the goals of ODS3 - Health and Wellbeing - when they were consulted on obstacles, challenges, and policy recommendations to meet the goals. The change of management of the government in December 2019, and the outbreak of the Covid-19 pandemic in 2020, broadened the investigation incorporating the analysis of the incumbent Minister of Health of the Nation on the 2030 Agenda, the study carried out and the current perspectives in the pandemic period. The results were analyzed from a comparative standpoint with a Brazilian study, which revealed that most experts agree on the country's potential to meet the goals of the 2030 Agenda. However, in the analysis of the new Minister of Health, there are "the paradoxes of the pandemic" that relate to the opportunity to empower the health system pursuant to the Covid-19 pandemic.
\end{abstract}

Key words 2030 Agenda, SDG, Sustainable development goals, Health and well-being, COVID-19
Resumen La Agenda 2030, una estrategia de la Organización de las Naciones Unidas (ONU) para promover un desarrollo humano global y sostenible capaz de satisfacer necesidades sociales básicas, aún se encuentra en sus etapas iniciales en la mayoría de los países de América del Sur. La presente investigación tuvo como objetivo consultar a un grupo de expertos en salud sobre las posibilidades de Argentina de cumplir con la Agenda 2030, en especial las metas del ODS3-Salud y Bienestar-, a su vez que se les consultó sobre los obstáculos, desafíos y recomendaciones de políticas para cumplir con las metas. El cambio de gestión del gobierno en diciembre de 2019, y el surgimiento de la pandemia COVID19 en el presente año, amplió la investigación incorporando el análisis del actual Ministro de Salud de la Nación sobre la Agenda 2030, el estudio realizado y las perspectivas actuales en el periodo de la pandemia. Los resultados fueron analizados en perspectiva comparada con un estudio brasilero, y mostraron que la mayoría de los expertos coincide en el bajo potencial del país para cumplir con las metas de la Agenda 2030. Sin embargo en el análisis del nuevo Ministro de salud surgen "las paradojas de la pandemia" que refieren a la oportunidad de fortalecer el sistema sanitario producto de la pandemia COVID-19.

Palabras clave Agenda 2030, Objeitvos del desarollo sustenibles, Salud y bienestar, Pandemia COVID-19 


\section{Introducción}

Transcurridos cinco años de su ratificación por 193 países, la Agenda 2030, una ambiciosa estrategia de la Organización de las Naciones Unidas (ONU) para promover un desarrollo humano global y sostenible capaz de satisfacer necesidades sociales básicas ${ }^{1}$, aún se encuentra en sus etapas iniciales en la mayoría de los países de América del Sur. Este lento ritmo de implementación de la Agenda 2030 tiende a empeorar debido a los impactos negativos de la pandemia COVID-19, sea por el aumento de la pobreza y la desigualdad, sea por el agravamiento de la crisis del multilateralismo y sus instituiciónes ${ }^{3}$, lo que dificulta la creación de una gobernanza global y de propuestas de financiamiento que apoyen efectivamente a los gobiernos nacionales en la implementación de políticas que permitan cumplir los 17 Objetivos de Desarrollo Sostenible (ODS), con sus 167 metas. Este escenario se torna aún más complejo cuando estos países deben recurrir a la ayuda de los organismos financieros internacionales que requieren a los países que adopten políticas para reducir el rol del Estado, concretizadas en privatizaciones, recorte del gasto público y las inversiones, reducción de la protección social y adopción de los superávits fiscales para garantizar el pago de las deudas contraídas.

Este es el caso de Argentina que entre 2016 y 2019 implementó políticas de retraimiento del Estado de sectores estratégicos, generando una profunda transformación económica y social que ha implicado la ampliación de las desigualdades, el acentuamiento de los procesos de exclusión, el aumento de la pobreza y de la desocupación, entre otros. En este marco, se puede destacar la adopción de -entre otras- tres medidas fundamentales: el fuerte cambio en los principales precios relativos de las variables económicas (salarios, insumos, tarifas y dólar), la quita o rebaja progresiva de retenciones a los productos primarios de exportación, y la desregulación y apertura del sector financiero. Todo esto ocurrió además en el marco de un aumento explosivo en la velocidad de endeudamiento externo del Estado durante esos mismos cuatro años ${ }^{2-3}$.

En el sector Salud, estas políticas implicaron el desfinanciamiento del sistema de salud, tanto en el subsector público como en la seguridad social y el sector privado. Este proceso es, en parte, producto de la devaluación que genera una estampida de los costos de insumos y medicamentos, de la inflación (...) y de la caída de la masa salarial, fuente de los recursos de la seguridad social, sumada a una visiblemente escasa disposición del Estado a evitar los aumentos de precios ${ }^{4} \mathrm{~A}$ esto se le suma un aspecto no menor, que implicó la degradación de la categoría del Ministerio de Salud de la Nación a rango de Secretaría del Ministerio de Desarrollo Social ${ }^{5}$.

En este escenario, el interrogante acerca de cuáles son las posibilidades de la Argentina de cumplir con la Agenda 2030, en especial las metas del ODS3-Salud y Bienestar, constituyó la pregunta realizada por una investigación internacional a un grupo de expertos en salud argentinos. Sus respuestas son la principal fuente de información en este artículo, cuyo objetivo principal consiste en analizar las percepciones de este grupo de expertos sobre las posibilidades de su país para cumplir con los objetivos del ODS3.

Para enriquecer este análisis, estas percepciones se trabajan en perspectiva comparada con las de expertos en salud en Brasil, donde se realizó el mismo estudio ${ }^{6}$. Cabe destacar que al finalizar la investigación, Argentina atravesó un proceso electoral que representó un recambio de las autoridades nacionales y el nuevo gobierno propuso cambiar radicalmente la dirección del país, especialmente sus políticas económicas y de salud. A los fines de profundizar el análisis, se decidió presentar las percepciones de los expertos al nuevo Ministro de Salud, y consultarle sobre las suyas. Desafortunadamente, en este proceso, la pandemia COVID-19 se convirtió en el centro de las preocupaciones mundiales, lo que llevó a los autores del artículo a incorporar el interrogante sobre cómo la pandemia podría interferir con el desafío de cumplir con la Agenda 2030 y la percepción que el Ministro de Salud tiene respecto a ello.

\section{Aspectos metodológicos}

El presente artículo fue elaborado a partir de los resultados del estudio 'Percepción de los especialistas en salud argentinos sobre los ODS y la Agenda 2030', desarrollado através de una asociación con el Centro de Estudios Estratégicos de Fiocruz (CEE-Fiocruz), del Departamento de Ciencias ENSP / FIOCRUZ y la Universidad Isalud, de Argentina, que conforman el Programa de Investigación Aplicada en Políticas de Salud (PIAPS).

Para este estudio, se consideraron como 'expertos en salud' a los autores principales de artículos en el campo de la salud pública, publicados en revistas indexadas en la base de datos Web of Science (WoS), desde septiembre de 2011 hasta 
septiembre de 2016 (mes en que se empezó la investigación).

La búsqueda de artículos se inició con la Web of Science (WoS), en la que se utilizaron las siguientes 'etiquetas de campo': (i) 'SU - área de investigación', en la que optamos por 'Salud pública, ambiental y ocupacional '; $\mathrm{Y}$ (ii) "CU - país / región”, donde se ingresó "Argentina”. En este proceso, se encontraron 505 artículos.

Para organizar estos artículos, se utilizó el software "Vantage Point", que extrajo la información del WoS y produjo una hoja de cálculo Excel con la siguiente información: título del artículo; autores; autor principal; correo electrónico; afiliación institucional del autor principal; y País. La selección de autores argentinos se realizó en este momento, lo que redujo el número de artículos a 266.

En este conjunto, el mismo autor podría llegar a tener más de un artículo seleccionado. Para evitar esto, identificamos la duplicación del correo electrónico de los autores principales, excluyéndolos. Luego de este proceso la muestra se redujo a 221 registros. También fue necesario verificar, entre los correos electrónicos seleccionados, aquellos que aún estaban vigentes. Para esto se utilizó un software de verificación de correo electrónico rápido, que llegó a 189 registros.

Un Instrumento diseñado por los investigadores fue enviado a estos 189 expertos durante el mes de enero de 2018 con un fecha límite de finalización y una devolución estipulada en 09/02/18. En ese intervalo de tiempo se enviaron tres recordatorios semanales. Respondieran al Instrumento 77 expertos $(40,7 \%$, una tasa alta para este tipo de investigación), que se constituyen en la muestra del presente trabajo.

$\mathrm{El}$ instrumento respondido por los expertos tomó como referencia la estructura utilizada por GlobeScan / SustainAbility Survey, en la encuesta 'Evaluating Progress Towards the Sustainable Development Goals', especialmente en lo que se refiere a la relación entre los ODS y la adopción de escalas tipo Likert.

El instrumento constó de 20 preguntas, 11 cerradas y nueve semiestructuradas, organizadas en tres partes: (a) "Perfil del encuestado"; (b) "Agenda 2030 y ODS"; y (c) "Medidas para cumplir las metas del ODS 3".

En las partes (1) y (2), ciertas preguntas presentaron una escala tipo Likert con una respuesta que varió del 1 al 5, donde 1 significaba 'muy bajo (a) potencial / importancia'; 2, "bajo potencial / importancia"; 3, "potencial / importancia media"; 4, "alto potencial / importancia"; y 5, "alto potencial / importancia".
Es importante señalar que dichas respuestas no se comparan con los resultados oficiales de Argentina en los ODS, lo cual implica una limitación del presente trabajo.

En el presente artículo, el Cuadros 1 y 3 utilizan datos que se refieren a los resultados obtenidos con esta escala. Para su elaboración, se decidió consolidar los datos mediante un promedio aritmético del valor numérico que representa las escalas asignadas por cada uno de los especialistas encuestados.

Entre las preguntas semi-estructuradas, el presente trabajo usa los datos que se refieren a las medidas que los expertos encuestados consideraron necesarias para el cumplimiento de cada una de las 9 metas del ODS 3. Estas medidas se describen aquí como "recomendaciones" de política.

El Cuadro 2 sistematiza las recomendaciones presentadas por los especialistas las cuales, debido a su gran cantidad, fueron clasificados y analizados a través de categorías temáticas. El Cuadro 3 muestra las respuestas de los expertos (usando la escala Likert mencionada anteriormente) a las recomendaciones propuestas por el Instrumento de Investigación.

$\mathrm{El}$ instrumento electrónico que fue enviado a los expertos tenía un formulario detallado de consentimiento informado (FCI). La programación digital de un instrumento de este tipo hizo posible que solamente pudieran realizar la encuesta los expertos que hubieran aceptado y enviado en FCI previamente. El FCI garantizó también el cumplimiento de los principios de bioética con respecto a la beneficencia, la no maleficencia, y la confidencialidad de los respondientes, ya que los profesionales que analizaron los datos registrados no tenían acceso a los nombres de los encuestados. Además, se explicó que las respuestas registradas solo se utilizarían para realizar trabajos académicos como este.

Una vez analizadas y sistematizadas las percepciones de los especialistas argentinos (i) fueron puestas en perspectiva con las de los especialistas brasileños que respondieron a investigaciones similares, como lo presentaron Moreira et $a^{6}$; y (ii) se realizó un entrevista con seis preguntas abiertas, en junio del 2020, al Ministro de Salud de Argentina, para conocer su análisis de los resultados, las políticas que el ministerio estaba desarrollando para los objetivos del ODS 3 y los efectos e impactos de la pandemia de COVID 19 sobre estas políticas y las condiciones de vida y salud de Argentina. En los siguientes apartados se presentan y discuten los datos aquí mencionados. 
Cuadro 1. Investigación "Argentina y la Agenda 2030 Percepciones de los especialistas en salud argentinos sobre el cumplimiento de los ODS: ODSs prioritarios para Argentina: ODSs que más contribuirían al logro del ODS 3; y potencial de Argentina para cumplir con los ODS. 2018. $\mathrm{n}=77$.

\begin{tabular}{|c|c|c|}
\hline $\begin{array}{c}\text { ODS que deberían ser prioridad } \\
\text { para Argentina. }(\%)\end{array}$ & $\begin{array}{c}\text { ODS que más contribuirían } \\
\text { para el ODS } 3(\%)\end{array}$ & $\begin{array}{l}\text { Potencial de Argentina para } \\
\text { cumplir con los ODS } \\
\text { (Media de escala de } 1 \text { a 5) }\end{array}$ \\
\hline ODS 1 -Fin a la pobreza $(19,0 \%)$ & ODS 4 -Educación $(20,61 \%)$ & $\begin{array}{l}\text { ODS } 5 \text {-Igualdad entre los } \\
\text { géneros (3) }\end{array}$ \\
\hline ODS 4 -Educación (17,5\%) & ODS 1 -Fin a la pobreza $(19,7 \%)$ & $\begin{array}{l}\text { ODS } 6 \text {-Disponibilidad de agua y } \\
\text { saneamiento }(2,8)\end{array}$ \\
\hline ODS 2 -Fin al hambre $(16,8 \%)$ & ODS 2 -Fin al hambre $(15,35 \%)$ & ODS 4 -Educación $(2,6)$ \\
\hline ODS 3 -Salud y bienestar $(11,36 \%)$ & $\begin{array}{l}\text { ODS } 6 \text {-Disponibilidad de agua y } \\
\text { saneamiento }(10,5 \%)\end{array}$ & $\begin{array}{l}\text { ODS } 17 \text {-Revitalizar la Alianza } \\
\text { Mundial para el Desarrollo } \\
\text { Sostenible }(2,5)\end{array}$ \\
\hline $\begin{array}{l}\text { ODS } 8 \text {-Crecimiento económico y } \\
\text { trabajo }(10,82 \%)\end{array}$ & $\begin{array}{l}\text { ODS } 8 \text {-Crecimiento económico y } \\
\text { trabajo }(9,6 \%)\end{array}$ & $\begin{array}{l}\text { ODS } 7 \text {-Energía segura y } \\
\text { sostenible }(2,4)\end{array}$ \\
\hline $\begin{array}{l}\text { ODS } 6 \text {-Disponibilidad de agua y } \\
\text { saneamiento }(5,19 \%)\end{array}$ & $\begin{array}{l}\text { ODS } 5 \text {-Igualdad entre los géneros } \\
(4,8 \%)\end{array}$ & $\begin{array}{l}\text { ODS } 14 \text {-Conservar los océanos y } \\
\text { mares }(2,3)\end{array}$ \\
\hline $\begin{array}{l}\text { ODS } 5 \text {-Igualdad entre los géneros } \\
(3,9 \%)\end{array}$ & $\begin{array}{l}\text { ODS } 10 \text {-Reducir la desigualdad en } \\
\text { y entre los países }(3,9 \%)\end{array}$ & ODS 3 -Salud y bienestar $(2,2)$ \\
\hline $\begin{array}{l}\text { ODS } 11 \text {-Ciudades y asentamientos } \\
\text { seguros } 0,039\end{array}$ & $\begin{array}{l}\text { ODS } 11 \text {-Ciudades y } \\
\text { asentamientos seguros }(3,5 \%)\end{array}$ & $\begin{array}{l}\text { ODS } 9 \text {-Infraesctructuras } \\
\text { resilientes, industrilaización e } \\
\text { inovación }(2,2)\end{array}$ \\
\hline $\begin{array}{l}\text { ODS } 16 \text {-Promover sociedades justas y } \\
\text { pacíficas }(3,9 \%)\end{array}$ & $\begin{array}{l}\text { ODS } 16 \text {-Promover sociedades } \\
\text { justas y pacíficas }(3,5 \%)\end{array}$ & $\begin{array}{l}\text { ODS } 12 \text {-Consumo y producción } \\
\text { sostenibles }(2,2)\end{array}$ \\
\hline $\begin{array}{l}\text { ODS } 13 \text {-Combatir el cambio climático } \\
(2,16 \%)\end{array}$ & $\begin{array}{l}\text { ODS } 12 \text {-Consumo y producción } \\
\text { sostenibles }(2,6 \%)\end{array}$ & ODS 2 -Fin al hambre $(2,1)$ \\
\hline $\begin{array}{l}\text { ODS } 10 \text {-Reducir la desigualdad en y } \\
\text { entre los países }(1,73 \%)\end{array}$ & $\begin{array}{l}\text { ODS } 13 \text {-Combatir el cambio } \\
\text { climático }(2,6 \%)\end{array}$ & $\begin{array}{l}\text { ODS } 11 \text {-Ciudades y } \\
\text { asentamientos seguros }(2,1)\end{array}$ \\
\hline $\begin{array}{l}\text { ODS } 15 \text {-Conservar los bosques y } \\
\text { detener la pérdida de biodiversidad } \\
(1,73 \%)\end{array}$ & $\begin{array}{l}\text { ODS } 15 \text {-Conservar los bosques y } \\
\text { detener la pérdida de biodiversidad } \\
(1,3 \%)\end{array}$ & $\begin{array}{l}\text { ODS } 13 \text {-Combatir el cambio } \\
\text { climático }(2,1)\end{array}$ \\
\hline $\begin{array}{l}\text { ODS } 9 \text {-Infraestructuras resilientes, } \\
\text { industrialización e innovación }(1,3 \%)\end{array}$ & $\begin{array}{l}\text { ODS } 7 \text {-Energía segura y sostenible } \\
(0,4 \%)\end{array}$ & $\begin{array}{l}\text { ODS } 15 \text {-Conservar los } \\
\text { bosques y detener la pérdida de } \\
\text { biodiversidad (2) }\end{array}$ \\
\hline $\begin{array}{l}\text { ODS } 12 \text {-Consumo y producción } \\
\text { sostenibles }(0,8 \%)\end{array}$ & $\begin{array}{l}\text { ODS } 9 \text {-Infraestructuras } \\
\text { resilientes, industrialización e } \\
\text { innovación }(0,4 \%)\end{array}$ & $\begin{array}{l}\text { ODS } 16 \text {-Promover sociedades } \\
\text { justas y pacíficas }(2)\end{array}$ \\
\hline $\begin{array}{l}\text { ODS } 17 \text {-Revitalizar la Alianza } \\
\text { Mundial para el Desarrollo Sostenible } \\
(0,4 \%)\end{array}$ & $\begin{array}{l}\text { ODS } 14 \text {-Conservar los océanos y } \\
\text { mares }(0,4 \%)\end{array}$ & $\begin{array}{l}\text { ODS } 8 \text {-Crecimiento económico } \\
\text { y trabajo }(1,9)\end{array}$ \\
\hline $\begin{array}{l}\text { ODS } 7 \text {-Energía segura y sostenible } \\
(0,0 \%)\end{array}$ & \multirow{2}{*}{$\begin{array}{l}\text { ODS } 17 \text {-Revitalizar la Alianza } \\
\text { Mundial para el Desarrollo } \\
\text { Sostenible }(0,4 \%)\end{array}$} & $\begin{array}{l}\text { ODS } 10 \text {-Reducir la desigualdad } \\
\text { en y entre los países }(1,9)\end{array}$ \\
\hline $\begin{array}{l}\text { ODS } 14 \text {-Conservar los océanos y } \\
\text { mares }(0,0 \%)\end{array}$ & & ODS 1 -Fin a la pobreza $(1,8)$ \\
\hline
\end{tabular}

Fuente: Elaboración propia en base al estudio 'Percepción de los especialistas en salud argentinos sobre los ODS y la Agenda 2030.'

\section{Percepciones de los expertos Argentinos sobre Agenda 2030 y ODS en el país}

\section{Perfil de los entrevistados}

La Tabla 1 presenta un breve perfil de los 77 expertos que respondieron al instrumento de in- vestigación: amplio predominio de las mujeres; la mayoría tiene entre 30 y 50 años (no hay menores de 30 años); mínimo de 10 años de experiencia profesional; nivel de conocimiento medio sobre la Agenda 2030; y percepción de que los ODS tienen alta importancia para guiar las políticas públicas. 
Cuadro 2. Investigación "Argentina y la Agenda 2030 Percepciones de los especialistas en salud argentinos sobre el cumplimiento de los ODS: recomendaciones de políticas para el cumplimiento de las metas del ODS 3. Clasificación Temática y distribución porcentual." 2018. N=77.

\begin{tabular}{|c|c|}
\hline Meta ODS & $\begin{array}{c}\text { Recomendaciones. Clasificación Temática } \\
\text { y distribución porcentual }\end{array}$ \\
\hline $\begin{array}{l}\text { Meta } 3.1 \text { - "Para 2030, reducir la tasa mundial de } \\
\text { mortalidad materna" }\end{array}$ & $\begin{array}{l}\text { Reducir la pobreza }(77,9 \%) \\
\text { Ampliar atención primaria }(69 \%) \\
\text { Legislación }(64 \%) \\
\text { Formación }(28 \%)\end{array}$ \\
\hline $\begin{array}{l}\text { Meta } 3.2 \text { - "Para 2030, poner fin a las muertes evitables de } \\
\text { recién nacidos y de niños menores de } 5 \text { años, logrando que } \\
\text { todos los países intenten reducir la mortalidad neonatal" }\end{array}$ & $\begin{array}{l}\text { Reducir la pobreza }(88,3 \%) \\
\text { Formación }(54 \%) \\
\text { Educación }(57 \%) \\
\text { Ampliar atención primaria }(76 \%) \\
\end{array}$ \\
\hline $\begin{array}{l}\text { Meta } 3.3 \text { - "Para 2030, poner fin a las epidemias del SIDA, } \\
\text { la tuberculosis, la malaria y las enfermedades tropicales } \\
\text { desatendidas y combatir la hepatitis, las enfermedades } \\
\text { transmitidas por el agua y otras enfermedades } \\
\text { transmisibles" }\end{array}$ & $\begin{array}{l}\text { Reducir la pobreza }(88,3 \%) \\
\text { Ampliar atención primaria }(79 \%) \\
\text { Investigación }(45 \%) \\
\text { Ampliar atención específica }(57 \%) \\
\text { Prevención y promoción }(63 \%)\end{array}$ \\
\hline $\begin{array}{l}\text { Meta } 3.4 \text { - "Para 2030, reducir en un tercio la mortalidad } \\
\text { prematura por enfermedades no transmisibles mediante la } \\
\text { prevención y el tratamiento y promover la salud mental y } \\
\text { el bienestar" }\end{array}$ & $\begin{array}{l}\text { Otros }(51 \%) \\
\text { Promoción de la salud y ambientes saludables(53) } \\
\text { Educación }(46 \%) \\
\text { Legislación }(46 \%) \\
\text { Ampliar atención primaria }(50 \%)\end{array}$ \\
\hline $\begin{array}{l}\text { Meta } 3.5 \text { - "Fortalecer la prevención y el tratamiento del } \\
\text { abuso de sustancias adictivas, incluido el uso indebido de } \\
\text { estupefacientes y el consumo nocivo de alcohol" }\end{array}$ & $\begin{array}{l}\text { Prevención y promoción (64\%) } \\
\text { Ampliar atención especifica }(48 \%) \\
\text { Otros }(53 \%) \\
\text { Legislación }(28,5 \%)\end{array}$ \\
\hline $\begin{array}{l}\text { Meta } 3.6 \text { - "Para 2020, reducir a la mitad el número de } \\
\text { muertes y lesiones causadas por accidentes de tráfico en el } \\
\text { mundo" }\end{array}$ & $\begin{array}{l}\text { Mejora de la Infraestructura }(62 \%) \\
\text { Transporte colectivo }(57 \%) \\
\text { Educación }(40 \%) \\
\text { Regulación }(78 \%) \\
\text { Punición }(27,27 \%)\end{array}$ \\
\hline $\begin{array}{l}\text { Meta } 3.7 \text { - "Para 2030, garantizar el acceso universal a los } \\
\text { servicios de salud sexual y reproductiva, incluidos los de } \\
\text { planificación de la familia, información y educación, y la } \\
\text { integración de la salud reproductiva en las estrategias y los } \\
\text { programas nacionales" }\end{array}$ & $\begin{array}{l}\text { Ampliar Atención primaria }(77,9 \%) \\
\text { Educación y comunicación }(71,4 \%) \\
\text { Promoción y Prevención }(70 \%) \\
\text { Genero }(74 \%)\end{array}$ \\
\hline $\begin{array}{l}\text { Meta } 3.8 \text { - "Lograr la cobertura sanitaria universal, en } \\
\text { particular la protección contra los riesgos financieros, el } \\
\text { acceso a servicios de salud esenciales de calidad y el acceso } \\
\text { a medicamentos y vacunas seguros, eficaces, asequibles y } \\
\text { de calidad para todos" }\end{array}$ & $\begin{array}{l}\text { Fortalecer cobertura salud }(80,5 \%) \\
\text { Protección Social }(55,8 \%) \\
\text { Políticas de precios de medicamentos }(62,3 \%) \\
\text { Ampliar cobertura medicamentos }(62,3 \%) \\
\text { Ampliar atención primaria }(59,4 \%)\end{array}$ \\
\hline $\begin{array}{l}\text { Meta } 3.9 \text { - "Para 2030, reducir sustancialmente el número } \\
\text { de muertes y enfermedades producidas por productos } \\
\text { químicos peligrosos y la contaminación del aire, el agua y } \\
\text { el suelo" }\end{array}$ & \begin{tabular}{|l} 
Fiscalización $(81,8 \%)$ \\
Regulación $(74 \%)$ \\
Protección laboral $(84 \%)$ \\
Educación $(70 \%)$ \\
Invertir en agroecología $(58,4)$
\end{tabular} \\
\hline
\end{tabular}

Fuente: Elaboración propia en base al estudio 'Percepción de los especialistas en salud argentinos sobre los ODS y la Agenda 2030.

En el Cuadro 1 se presentan las percepciones de los expertos sobre los ODS que deberían ser priorizados por el país. Los tres más importantes son, para ellos, el ODS 1 (Erradicación de la po- breza), el ODS 4 (Educación), y el ODS 2 (Erradicación del hambre). Estos ODS son, también, los que más contribuyen para el logro del ODS 3 (el cuarto más importante). 
Cuadro 3. Investigación "Argentina y la Agenda 2030 Percepciones de los especialistas en salud argentinos sobre el cumplimiento de los ODS: recomendaciones de políticas para el cumplimiento de las metas del ODS 3 y su grado de importancia (Media de escala de 1 a 5) según los especialistas consultados.” 2018. n=77.

\begin{tabular}{|c|c|c|}
\hline Meta ODS & Recomendaciones & $\begin{array}{c}\text { Grado de } \\
\text { Importancia }\end{array}$ \\
\hline $\begin{array}{l}\text { Meta } 3.1 \text { - "Para } 2030, \\
\text { reducir la tasa mundial de } \\
\text { mortalidad materna" }\end{array}$ & $\begin{array}{l}\text { Reducir la pobreza } \\
\text { Ampliar y jerarquizar los servicios prenatales en la Atención } \\
\text { Primaria } \\
\text { Despenalizar el aborto } \\
\text { Formar y aumentar la cantidad de enfermeras y obstétricas } \\
\text { Reducir los índices de cesárea }\end{array}$ & $\begin{array}{l}4,6 \\
4,4 \\
4,2 \\
3,8 \\
2,7\end{array}$ \\
\hline $\begin{array}{l}\text { Meta } 3.2 \text { - "Para } 2030, \\
\text { poner fin a las muertes } \\
\text { evitables de recién nacidos } \\
\text { y de niños menores de } 5 \\
\text { años, logrando que todos } \\
\text { los países intenten reducir } \\
\text { la mortalidad neonatal" }\end{array}$ & $\begin{array}{l}\text { Reducir la pobreza } \\
\text { Aumentar la calificación en el cuidado al recién nacido y al niño } \\
\text { en la Atención Primaria } \\
\text { Reducir el número de embarazos no deseados en la adolescencia } \\
\text { Ampliar la oferta de camas de servicios neonatal y pediátrico } \\
\text { Aumentar la oferta de guarderías y de espacios de desarrollo } \\
\text { infantil }\end{array}$ & $\begin{array}{l}4,8 \\
4,3 \\
4,3 \\
3,7 \\
3,5\end{array}$ \\
\hline $\begin{array}{l}\text { Meta } 3.3 \text { - "Para } 2030, \\
\text { poner fin a las epidemias } \\
\text { del SIDA, la tuberculosis, la } \\
\text { malaria y las enfermedades } \\
\text { tropicales desatendidas y } \\
\text { combatir la hepatitis, las } \\
\text { enfermedades transmitidas } \\
\text { por el agua y otras } \\
\text { enfermedades transmisibles }\end{array}$ & $\begin{array}{l}\text { Reducir la pobreza } \\
\text { Alcanzar la cobertura universal de la Atención Primaria } \\
\text { Aumentar la cobertura de saneamiento básico } \\
\text { Ampliar las acciones de prevención de la Tuberculosis y el SIDA } \\
\text { con terapias de profilaxis } \\
\text { Ampliar las pruebas de VIH e iniciar el tratamiento precoz } \\
\text { Ampliar la cobertura de vacunación para las hepatitis } \\
\text { Ampliar la investigación y el desarrollo de vacunas y } \\
\text { medicamentos }\end{array}$ & $\begin{array}{l}4,8 \\
4,7 \\
4,6 \\
4,5 \\
4,3 \\
4,3 \\
4\end{array}$ \\
\hline $\begin{array}{l}\text { Meta } 3.4 \text { - "Para } 2030, \\
\text { reducir en un tercio la } \\
\text { mortalidad prematura } \\
\text { por enfermedades no } \\
\text { transmisibles mediante } \\
\text { la prevención y el } \\
\text { tratamiento y promover la } \\
\text { salud mental y el bienestar" }\end{array}$ & $\begin{array}{l}\text { Reducir los índices de obesidad entre niños, adolescentes y adultos } \\
\text { Reducir las diferentes formas de violencia } \\
\text { Ampliar la red de atención a la salud mental } \\
\text { Ampliar las políticas de promoción de la salud relacionadas con } \\
\text { los estilos de vida } \\
\text { Reducir los niveles de tabaquismo } \\
\text { Ampliar la regulación de alimentos industrializados }\end{array}$ & $\begin{array}{l}4,3 \\
4,3 \\
4,2 \\
4,2 \\
4,2 \\
4,1\end{array}$ \\
\hline $\begin{array}{l}\text { Meta } 3.5 \text { - "Fortalecer la } \\
\text { prevención y el tratamiento } \\
\text { del abuso de sustancias } \\
\text { adictivas, incluido el uso } \\
\text { indebido de estupefacientes } \\
\text { y el consumo nocivo de } \\
\text { alcohol" }\end{array}$ & $\begin{array}{l}\text { Ampliar la prevención del abuso de alcohol } \\
\text { Ampliar el acceso a terapias de desintoxicación no punitivas } \\
\text { Ampliar las políticas de reducción de daños } \\
\text { Legalizar la marihuana } \\
\text { Legalizar otras drogas }\end{array}$ & $\begin{array}{l}4,5 \\
4,3 \\
4,2 \\
3,3 \\
2,2\end{array}$ \\
\hline
\end{tabular}

La percepción de los encuestados sobre el potencial de Argentina para cumplir con los ODS es negativa. Las puntuaciones de los 17 objetivos variaron entre 1.8 ('bajo' potencial) y 3.0 ('medio' potencial). El ODS 5 (igualdad de género) y el ODS 6 (agua potable y saneamiento) son los que tienen mejores posibilidades de cumplimiento en un rol en que el ODS 3 figura en la 8ava posición. En el otro extremo de esta lista, el ODS 10 (reducción de las desigualdades) y el
ODS 1 (Erradicación de la pobreza) tienen el menor potencial de cumplimiento.

Con el foco en ODS 3 (Salud y Bienestar), el Gráfico 1 presenta los factores que los expertos eligieron como principales problemas para su logro, con destaque para las "políticas de ajuste del gasto público", la "mala calidad de la gestión en salud" y la "baja integración de los políticas públicas sectoriales". 
Cuadro 3. Investigación “Argentina y la Agenda 2030 Percepciones de los especialistas en salud argentinos sobre el cumplimiento de los ODS: recomendaciones de políticas para el cumplimiento de las metas del ODS 3 y su grado de importancia (Media de escala de 1 a 5) según los especialistas consultados.” 2018. n=77.

\begin{tabular}{|c|c|c|}
\hline Meta ODS & Recomendaciones & $\begin{array}{c}\text { Grado de } \\
\text { Importancia }\end{array}$ \\
\hline \multirow{6}{*}{$\begin{array}{l}\text { Meta } 3.6 \text { - "Para 2020, } \\
\text { reducir a la mitad el } \\
\text { número de muertes y } \\
\text { lesiones causadas por } \\
\text { accidentes de tráfico en el } \\
\text { mundo" }\end{array}$} & Mejorar la calidad de calles y rutas & 4,4 \\
\hline & $\begin{array}{l}\text { Ampliar las políticas de movilidad urbana que incentiven el uso } \\
\text { del transporte colectivo }\end{array}$ & 4,3 \\
\hline & $\begin{array}{l}\text { Aumentar la obligación de equipar la flota de automóviles con } \\
\text { elementos de seguridad }\end{array}$ & 4,1 \\
\hline & $\begin{array}{l}\text { Regular la propaganda de autos para no estimular la conducción } \\
\text { agresiva }\end{array}$ & 3,9 \\
\hline & Implementar la Ley Seca en todo el territorio nacional & 3,6 \\
\hline & Comprometer y responsabilizar a las empresas automotrices & 3,6 \\
\hline \multirow{4}{*}{$\begin{array}{l}\text { Meta } 3.7 \text { - "Para 2030, } \\
\text { garantizar el acceso } \\
\text { universal a los servicios } \\
\text { de salud sexual y } \\
\text { reproductiva, incluidos } \\
\text { los de planificación de la } \\
\text { familia, información y } \\
\text { educación, y la integración } \\
\text { de la salud reproductiva } \\
\text { en las estrategias y los } \\
\text { programas nacionales" }\end{array}$} & $\begin{array}{l}\text { Alcanzar la cobertura universal de la Atención Primaria con } \\
\text { componentes de salud sexual y reproductiva }\end{array}$ & 4,7 \\
\hline & Ampliar las acciones de educación y comunicación masiva & 4,6 \\
\hline & $\begin{array}{l}\text { Implementar políticas específicas para adolescentes vinculadas } \\
\text { al deporte, educación, cultura y otras que incorporen educación } \\
\text { sobre salud sexual y reproductiva }\end{array}$ & 4,6 \\
\hline & $\begin{array}{l}\text { Incorporar discusiones sobre género, sexualidad, misoginia, } \\
\text { machismo, homofobia y preconceptos en las escuelas }\end{array}$ & 4,4 \\
\hline \multirow{5}{*}{$\begin{array}{l}\text { Meta } 3.8 \text { - "Lograr la } \\
\text { cobertura sanitaria } \\
\text { universal, en particular } \\
\text { la protección contra los } \\
\text { riesgos financieros, el } \\
\text { acceso a servicios de salud } \\
\text { esenciales de calidad y el } \\
\text { acceso a medicamentos y } \\
\text { vacunas seguros, eficaces, } \\
\text { asequibles y de calidad } \\
\text { para todos" }\end{array}$} & Alcanzar la cobertura universal de la Atención Primaria & 4,7 \\
\hline & $\begin{array}{l}\text { Ampliar las políticas públicas de protección social, como } \\
\text { transferencias de ingresos, vivienda y educación }\end{array}$ & 4,5 \\
\hline & Ampliar las políticas de reducción de precios de medicamentos & 4,4 \\
\hline & Ampliar la distribución gratuita de medicamentos & 4,4 \\
\hline & $\begin{array}{l}\text { Mejorar los mecanismos de regulación del acceso a los servicios } \\
\text { hospitalarios y de alta complejidad en el ámbito público y privado }\end{array}$ & 4,3 \\
\hline \multirow{5}{*}{$\begin{array}{l}\text { Meta } 3.9 \text { - "Para } 2030, \\
\text { reducir sustancialmente } \\
\text { el número de muertes } \\
\text { y enfermedades } \\
\text { producidas por productos } \\
\text { químicos peligrosos y la } \\
\text { contaminación del aire, el } \\
\text { agua y el suelo" }\end{array}$} & $\begin{array}{l}\text { Ampliar el control sobre actividades de extracción y de industrias } \\
\text { contaminantes }\end{array}$ & 4,8 \\
\hline & Aumentar el control sobre el uso de agroquímicos & 4,7 \\
\hline & Ampliar la protección a trabajadores expuestos a riesgo laboral & 4,6 \\
\hline & Ampliar el uso de energía limpia y renovable & 4,5 \\
\hline & $\begin{array}{l}\text { Ampliar las políticas de incentivo a la agricultura orgánica y a la } \\
\text { agricultura familiar }\end{array}$ & 4,3 \\
\hline
\end{tabular}

Fuente: Elaboración propia en base al estudio 'Percepción de los especialistas en salud argentinos sobre los ODS y la Agenda 2030'

\section{Medidas para cumplir las metas del ODS 3}

Considerando distintas maneras a partir de las cuales se podrían superar estas dificultades y pensando en qué dirección debería seguir Argentina para alcanzar el cumplimiento de los ODS y hacer efectiva la Agenda 2030, la investigación intentó plantear a los especialistas un conjunto de medidas, políticas y acciones que, en este trabajo, denominaremos "recomendaciones de política".

El Cuadro 2 sistematiza las 47 recomendaciones de políticas que los expertos señalaron que debían implementarse para el cumplimiento del ODS 3, separadas según las nueve metas propuestas de este Objetivo. Se destacan: 'reducción de la pobreza' (metas 3.1; 3.2 y 3.3); 'Ampliar atención 
Tabla 1. Investigación "Argentina y la Agenda 2030 Percepciones de los especialistas en salud argentinos sobre el cumplimiento de los ODS . Perfil de los expertos consultados. 2018. (n=77)

\begin{tabular}{llcc}
\hline \multicolumn{1}{c}{ Perfil } & \multicolumn{1}{c}{ Características } & Números absolutos & Porcentaje \\
\hline Franja Etaria & Hasta 30 años & 0 & $0,0 \%$ \\
& 31 a 40 años & 24 & $31,0 \%$ \\
& 41 a 50 años & 24 & $31,0 \%$ \\
& 51 a 60 años & 16 & $21,0 \%$ \\
& 61 a 70 años & 10 & $13,0 \%$ \\
& Más de 71 años & 3 & $4,0 \%$ \\
Tiempo de experiencia & 5 a 10 años & 17 & $22,0 \%$ \\
profesional & 11 a 20 años & 34 & $44,0 \%$ \\
Sexo & 21 a 30 años & 16 & $20,7 \%$ \\
Nivel de conocimiento sobre & Más de 30 años & 10 & $12,9 \%$ \\
ODS/Agenda 2030 & Femenino & 58 & $75,0 \%$ \\
& Masculino & 16 & $25,0 \%$ \\
& Medio & 32 & $41,5 \%$ \\
& Alto & 15 & $19,4 \%$ \\
Importancia de los ODS para & Muy Alto & 4 & $5,2 \%$ \\
orientar las políticas públicas & Muy Bajo & 2 & $2,6 \%$ \\
& Bajo & 8 & $10,4 \%$ \\
& Muy Alto & 23 & $29,9 \%$ \\
\hline
\end{tabular}

Fuente: Elaboración propia en base al estudio 'Percepción de los especialistas en salud argentinos sobre los ODS y la Agenda 2030.'

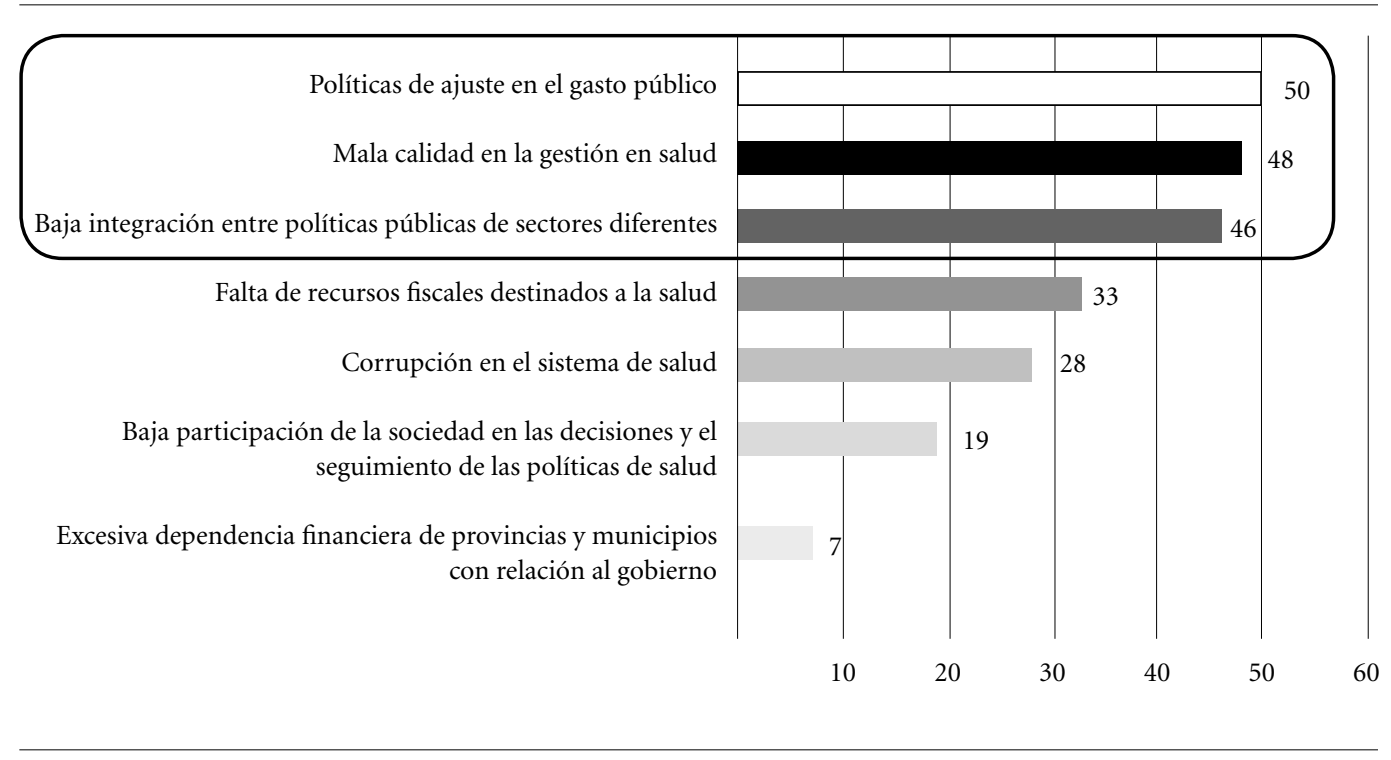

Gráfico 1. Investigación "Argentina y la Agenda 2030 - Percepciones de los especialistas en salud argentinos sobre el cumplimiento de los ODS: Factores que impiden el logro del ODS 3 en porcentaje". 2019. N=77

Fuente: Elaboración propia en base al estudio 'Percepción de los especialistas en salud argentinos sobre los ODS y la Agenda 2030.'

básica’ (metas 3.3; 3.2; 3.3; 3.4; 3.7 y 3.8); y 'Educación' (políticas que apuntan a población en general, en las metas $3.2 ; 3.4 ; 3.6 ; 3.7$ y 3.9 ).
De manera resumida podemos resaltar el alto grado de importancia que los encuestados asignaron a estas primeras 47 recomendacio- 
nes. Si consideramos la escala propuesta, que va desde 1 "bajo nivel potencial" hasta 5 "alto nivel potencial", podemos ver que 38 de las 47 políticas $(81,0 \%)$ puntúan como de "alta importancia (entre 4 y 4,9 puntos); 7 respuestas (15,0\%) puntúan como de importancia razonable (entre 3 y 3,9 puntos) y solamente 2 respuestas $(4,0 \%)$ puntúan en el promedio general como de baja importancia (2 a 2,9 puntos).

En el Cuadro 3 se muestran, para cada meta del ODS 3, estos resultados a partir de las 47 recomendaciones de política más seleccionadas por los encuestados. En esta tabla se puede observar un agrupamiento temático de las respuestas (realizado ex post), que considera también la valoración que los expertos consultados hicieron de estas recomendaciones.

Agrupando temáticamente las respuestas que pueden pensarse de manera transversal a todas las metas se observa que la reducción de la pobreza y la ampliación de la cobertura universal de APS son las políticas que más aparecen, sobre todo con relación a las metas 3.1 y 3.2 vinculadas con la salud materno infantil.

De la misma forma, aparecen con mucha fuerza las políticas de promoción y prevención de la salud y los cuidados preventivos. Este tipo de políticas que pueden ir desde la incorporación de discusiones sobre género, sexualidad, misoginia, machismo, homofobia y preconceptos en las escuelas hasta la ampliación de las acciones de prevención de la tuberculosis y el SIDA, se distribuye con igual importancia a lo largo de las distintas metas.

También se observan como importantes, según los encuestados, dos recomendaciones de política relacionadas con la meta 3.9 vinculada a la salud ambiental, estas son la ampliación del control sobre actividades de extracción y de industrias contaminantes y también el control sobre el uso de agroquímicos.

En el otro extremo las dos recomendaciones de política que menos consideración obtuvieron fueron las referidas a la reducción de los índices de cesárea en la meta 3.1 (Reducir la tasa de mortalidad materna) y la referida a la legalización de otras drogas en la meta (3.5 Fortalecer la prevención y el tratamiento del abuso de sustancias adictivas, incluido el uso indebido de estupefacientes y el consumo nocivo de alcohol)

\section{Análisis de las percepciones de los expertos argentinos y comparación con los expertos brasileños}

El primer aspecto a destacar se refiere a las percepciones sobre prioridades: los argentinos y brasileños tienen una opinión muy similar y consideran el fin de la pobreza (ODS 1) y el hambre (ODS 2) y la educación (ODS 4) como las prioridades para ambos países, $y$, también, los ODSs que más contribuyen para el logro de ODS 3.

Los expertos argentinos consideran que el País tiene pocas potencialidades de cumplir con la Agenda 2030, situación que es similar entre los expertos brasileños.

Según autores de los dos países ${ }^{7-8}$ este descrédito está relacionado con el escenario político-económico, marcado por el impacto social de la austeridad fiscal, la reducción del papel del Estado, el ataque a las políticas de protección social y el predominio del capital financiero.

Esto se refleja en la percepción de que los procesos de salud deben ser entendidos como el resultado de determinantes mucho más complejos que implican la correlación de componentes individuales, económicos, sociales, culturales y ambientales. En este sentido, para mejorar los procesos y resultados de salud deben mejorar los indicadores socioeconómicos ${ }^{9-11}$.

Según la percepción de los especialistas argentinos, las "políticas de ajuste en el gasto público", "la mala calidad de la gestión de la salud" y la "baja integración entre políticas públicas de diferentes sectores" son, aislados, los tres factores principales que impiden que Argentina cumpla ODS 3. Agrupando los factores presentados en el Gráfico 1, se concluye que los expertos considerarán la "Gestión” como el factor más importante $(69.0 \%)$, superando los "Recursos financieros" $(31,0 \%)$.

Como indica Tobar ${ }^{12}$ todo sistema de salud puede ser pensado como la articulación de un modelo de gestión (político), un modelo de financiamiento (económico) y un modelo de atención (técnico), cada uno de estos ejes puede tener una supremacía sobre los demás a la hora de planificar una política pública. En los últimos procesos de reformas del sector salud se ha priorizado, en Argentina, el debate sobre los modelos de financiamiento y se ha tendido a dejar de lado la consideración de los modelos de gestión y de atención. En este marco surge la necesidad de rescatar el debate acerca de la gestión de políticas públicas, poniendo la centralidad en la intervención estatal y la política y cuestionando la 
mercantilización de la protección social por los efectos de inequidad y desigualdad, a la vez que resituar el debate sobre la universalidad de las políticas sociales ${ }^{13-14}$.

Estos datos indican que, si los especialistas argentinos y brasileños convergen en el descrédito con el cumplimiento del ODS 3 por parte de sus países, los factores que motivan esta percepción difieren considerablemente, ya que, para los brasileños, la 'corrupción' y la 'baja participación de la sociedad' (que, para los argentinos, aparecen como los factores quinto y sexto) superan el ajuste fiscal y la falta de intersectorialidad. Lo mismo sucede cuando nos enfocamos en las dos categorías agregadas, en las cuales los "Recursos financieros" alcanzan, para los brasileños, el 83.4\%, frente al 26.6\% para la "Gestión".

El análisis de el cuadro 2 muestra que, para los especialistas argentinos, en términos generales, la "Reducción de la pobreza" es la recomendación política más importante, en particular para lograr los objetivos relacionados con la mortalidad materna e infantil y el fin de epidemias como el SIDA y la tuberculosis. También se hace hincapié en la "expansión de la atención primaria" y la "educación". La relación entre la reducción de la pobreza y las enfermedades ha sido objeto de muchos estudios en la Argentina ${ }^{15-16}$, y en este sentido, hay consenso de que las políticas sanitarias deben articularse con políticas públicas que permitan mejorar las condiciones socioeconómicas $^{17-18}$.

También en este sentido, las percepciones de argentinos y brasileños no convergen completamente, ya que, para los brasileños, "Educación" es la recomendación más importante, excepto en los objetivos 3.2 (es el segundo más importante), 3.8 y 3.9. Es posible considerar que esta divergencia está relacionada con las diferencias de acceso y permanencia en la escuela, que en Argentina ha tenido mejores indicadores que Brasil durante muchos años.

El Cuadro 3 muestra la evaluación de los expertos argentinos de un conjunto de 47 recomendaciones de políticas propuestas por la encuesta. De este total, 39 recomendaciones fueron calificadas como de "alta importancia", mientras que siete tienen "importancia razonable" y 2 " baja importancia".

Cuando estas respuestas se ponen en perspectiva con las de los expertos brasileños, la mayoría de ellas muestran una convergencia considerable. Sin embargo, existen desacuerdos importantes: (i) para la reducción de la mortalidad materna, la despenalización del 'aborto' es más valorada por los argentinos, en contraste con la reducción en las tasas de cesárea; (ii) para la reducción de la mortalidad infantil, la ampliación de la oferta de camas neonatales y pediátricas y de centros y espacios de desarrollo infantil recibe una calificación "alta" por los argentinos y "media" por los brasileños; (iii) para reducir las muertes por accidentes de tránsito, la implementación de la ley seca en el territorio nacional es calificada por los brasileños como "alta", mientras que los argentinos la perciben como "media".

Entre las recomendaciones evaluadas como de alta importancia, se hace hincapié en 'Reducir la Pobreza' (principal para los objetivos 3.1, 3.2 y 3.3) y las relacionadas con 'Atención primaria' (la cobertura universal es la primera para las metas 3.7 y 3.8 , segunda en la meta 3.3 , además de ser incluido en otros objetivos, como, por ejemplo, atención primaria para el recién nacido, en la 3.2).

Las investigaciones basadas en el análisis de políticas tienen como objetivo discutir los resultados y las recomendaciones con el gobierno, buscando aportar evidencia para la toma de decisiones. Este artículo también se enmarca en esta línea de investigaciones. Como, a lo largo de 2019, Argentina pasó por un proceso electoral, se decidió entrevistar al Ministro de Salud del nuevo gobierno (independientemente de quién fuera) para interrogarlo sobre el escenario planteado por la percepción de los especialistas argentinos y sobre las políticas que se implementarían para cumplir con la Agenda 2030.

\section{Discusión de las percepciones de los expertos argentinos con el Ministro de Salud: paradojas de la pandemia COVID-19}

En diciembre de 2019, el presidente Alberto Fernández asumió el nuevo gobierno en la Argentina. Elegido en la primera vuelta y bajo los auspicios de una fuerte oposición al gobierno anterior, nombró a Ginés Gonzales García como Ministro de Salud, un puesto que había ocupado anteriormente. La entrevista con el Ministro, en junio del 2020, coincidió con el surgimiento de la pandemia COVID19, que requería que se incluyeran preguntas sobre cómo la pandemia afectaría a Argentina y las políticas destinadas a cumplir la Agenda 2030.

El Ministro coincidió con la mayoría de las percepciones críticas y negativas expresada por los expertos argentinos. Según el, para afrontar los retos como los apuntados por los expertos, la primera medida fue la reversión de la desjerar- 
quización del Ministerio de Salud (que ya había abogado en la campaña electoral) para ...recuperar la autoridad del Ministerio nacional para coordinar y conducir al resto del sistema, y en concertar un plan federal plurianual que promueva una mayor integración de los sistemas de salud.

Al mismo tiempo fue implementada la "Ley de Emergencia Sanitaria" con el objetivo de recuperar el sistema de salud que el Ministro describió como "al borde del colapso". Dicha medida, considera, tendrá un impacto directo en el acceso a los servicios y la reducción de las inequidades, contribuyendo al cumplimiento de la Agenda 2030, a través de medidas como (i) garantizar la cobertura de vacunas; (ii) relanzar el programa 'Remediar' que distribuye medicamentos gratuitamente en todo el país en los centros de atención primaria; (iii) consensuar con distintos actores una ley para el aborto legal; (iv) ampliar la integración de la salud reproductiva en los programas nacionales; (v) ampliar las campañas para prevenir y controlar el Dengue; y con estas medidas (vii) reducir los gastos del bolsillo de las familias.

La Pandemia COVID-19 surgió en un momento en que estas políticas estaban siendo implementadas. Según el Ministro: (...)la pandemia nos puso en un escenario inédito, no sólo en Argentina sino en todo el mundo(...) el mundo que viene no va a ser igual(...) cambiará el sistema de salud(...) los países vamos a ser más pobres, al menos inicialmente. Los problemas de salud más relevantes de los ODS son las patologías que dependen de los estilos de vida y de la pobreza(...) si la pobreza se agrava, los objetivos de ODS quedarán más lejos de ser logrados.

Sin embargo, al analizar el escenario, el Ministro presentó una percepción distinta en la que señala que la lucha contra la Pandemia tiene el potencial de producir cambios en el sistema de salud, que llevarían a una visión más optimistas con respecto al cumplimiento de la Agenda 2030. En este artículo, los elementos que componen esta percepción del Ministro serán denominadas "paradojas de la pandemia". En este sentido, el hecho de que la pandemia haya llevado a concentrar parte de las politicas públicas en el sector de salud podría contribuir en el mediano y largo plazo a mejorar las metas propuestas en los ODS.

La primera de estas paradojas está relacionada a los servicios de salud:

(...) la pandemia nos llevó a concentrar mucho tiempo de gestión y recursos para recuperar rápidamente la capacidad de atención del sistema de salud. (...) la cantidad de camas de terapia en el sector público aumentó un $80 \%$ en los dos últimos meses. (...) en pocos meses el Estado debió invertir muy fuertemente en reequipar el sistema, principalmente en el sector público, y en equilibrar las coberturas de los distintos subsistemas. (...) si logramos evitar que el sistema colapse por el COVID-19, quedará una capacidad instalada que permite mirar los años que vienen de manera más optimista, porque todos los inviernos el sistema quedaba desbordado y aumentaba fuertemente la mortalidad por diversas causas, principalmente respiratorias.

Una segunda paradoja se refiere a la integralidad de los subsistemas de salud:

(...) aumentó la capacidad de trabajar coordinada e integradamente con el sector privado $y$ las obras sociales. El sector privado y el de la seguridad social comparten hoy con el sector público información crítica que antes se desconocía. Esa información es determinante de las posibilidades de avanzar en la integración de los sistemas de salud.

En el mismo tono, la articulación federal también forma parte de este rol de paradojas:

(...) se pudo llegar a rápidos acuerdos y a una eficaz coordinación operativa con los gobiernos provinciales, independientemente del signo de los partidos politicos gobernantes.

Finalmente, la importante tarea de hacer llegar a la población informaciones correctas, con base en la ciencia y de calidad:

(...) se aprovecha el amplio espacio que está teniendo salud en los medios de comunicación para realizar campañas de prevención de otras enfermedades como el dengue, las enfermedades coronarias o la promoción de prácticas saludables.

En relación con los ODS, el Ministro considera que las desigualdades sociales y regionales son las principales dificultades de Argentina para cumplir con la Agenda 2030:

...hay que tener en cuenta que la Argentina es un país con una severa desigualdad socioeconómica preexistente, $y$ por eso tenemos que idear politicas ambiciosas para hacer que en salud esa desigualdad disminuya, concentrándonos en quienes más necesitan de la protección del Estado. Por ejemplo, hay varias metas de los ODS que fácilmente se alcanzarán en algunas jurisdicciones y en otras no: uno de nuestros objetivos prioritarios al frente del Ministerio es trabajar para reducir esas brechas.

\section{Consideraciones finales}

Los resultados y análisis trabajados aquí, muestran que la percepción del bajo potencial para el cumplimiento de los ODS prevalece tanto 
entre los expertos Argentinos como entre los expertos Brasileños. Esta percepción negativa tiene relación no solamente con los problemas en el sector de la salud, sino también con las dificultades que ambos países afrontan para superar la pobreza, el hambre y la desigualdad.

En este escenario, las "paradojas de la pandemia" señaladas por el Ministro de Salud de Argentina reflejan, como él mismo reconoce, una visión optimista cuya realización deberá analizarse mediante nuevas investigaciones sobre la pandemia COVID-19, el cumplimiento de la Agenda 2030, la mejora del sistema de salud y las condiciones de vida de la población.

No obstante, el análisis en una perspectiva crítica de las percepciones y recomendaciones de los expertos y de ciertos aspectos de las paradojas de la pandemia, indica un conjunto importante de medidas que tienen poder efectivo para enfrentar los problemas tradicionales del sistema de salud, agravados por la pandemia.

\section{Colaboradores}

M Fontela, M Monsalvo y S Beltrame participaron en en el diseño y coordinación del estudio, recolección de datos y interpretación de los datos. E Kastrup Bittencourt e Câmara J Mendes Ribeiro y M Rasga Moreira participaron en en el diseño y coordinación del estudio, recolección de datos y interpretación de los datos y la redacción del manuscrito. Todos los autores revisaron y aprobaron la versión final del artículo.
Este artículo termina destacando cuatro de estas medidas: (i) valorar la información correcta y con base científica como guía para la política de salud y como contrapunto a las "Fake News". En este proceso, debe priorizarse la comunicación con la sociedad, siendo los medios responsables de difundir la información correcta; (ii) articulación entre los sistemas de salud públicos y privados, evitando privilegios para algunos y sobrecargas para otros; (iii) priorización de las posiciones democráticas y republicanas por parte de los funcionarios del gobierno, quienes, a pesar de sus partidos y orientaciones políticas, deben actuar como representantes de una sociedad que debe llegar a acuerdos y consensos en nombre del bienestar de la población; $y$, lo más importante de todo, (iv) una inversión estatal masiva en salud, protección social y sistemas de creación de empleo. 


\section{Referencias}

1. Organização das Nações Unidas (ONU). Transformando o nosso mundo: a agenda 2030 para o desenvolvimento sustentável. Resolução A/RES/70/1. Nova Iorque: ONU; 2015. [acceso 2020 Nov 08]. Disponible en: https://nacoesunidas.org/wp-content/uploads/2015/10/agenda2030-pt-br.pdf

2. Canelo P, Castellani A, Gentile J. El gobierno de los CEOs. Equivalencia entre elites económicas y políticas en el gabinete de Mauricio Macri (2015-2018) Voces en el Fénix. Vol. 8. Lugar: Buenos Aires; 2018 p. 93-97.

3. Manzanelli, Daniel P, Basualdo, Marcelo E. La deuda externa de Macri en perspectiva histórica; Universidad de Buenos Aires. Facultad de Ciencias Económicas. Plan Fénix; Voces en el Fénix. 2017; p. 18-25.

4. García GG, Tobar, Federico. Desventajas de no tener un Ministerio de Salud. Rev Isalud 2018; 13(64).

5. Decreto 802/2018. Mediante el Decreto Decreto 802/2018, en septiembre de 2018 se modificó la Ley de Ministerios y el Ministerio de Salud paso a convertirse en una secretaria de gobierno dependiente del Ministerio de Desarrollo Social. 2018.

6. Rasga MM, Kastrup E, Ribeiro JM, Carvalho AI, Braga AP. O Brasil rumo a 2030? Percepções de especialistas brasileiros(as) em saúde sobre o potencial de o País cumprir os ODS Brazil heading to 2030. Saude Debate 2019; 43(n. spe. 7):22-35.

7. Grimson A. Entrevista. Rev Conciencia Social 2016; 1:122-126.

8. Canelo P, Castellani A. Informe de investigación no 1 . Perfil sociológico de los miembros del gabinete inicial del presidente Mauricio Macri, Observatorio de las elites argentinas, IDAES-UNSAM. 2016. $\mathrm{p} 38$.

9. Alazraqui M, Spinelli H, Guevel C, Mota E. Desigualdades en salud y desigualdades sociales. En: Alazraqui M, Spinelli H. Desigualdades en Salud en el nivel local/municipal. Remedios de Escalada: Ediciones de la UNLa; 2008. p. 189-208.

10. Vega A, Zunino MG, Spinelli H. Espacio geográfico y epidemiología. Geoprocesamiento y estudio de las desigualdades en salud. En: Alazraqui M, Spinelli $H$. Desigualdades en Salud en el nivel local/municipal. Remedios de Escalada: Ediciones de la UNLa; 2008. p. 49-65.
11. Spinelli H. Condiciones de Salud y Desigualdades Sociales: historias de iguales, desiguales y distintos. En: Minayo MC, Coimbra C, organizadores. Críticas e Atuantes: ciências sociais e humanas em saúde na América Latina. Rio de Janeiro: Editora Fiocruz; 2005. p. 315-332.

12. Tobar F. Modelos de gestión en salud. Buenos Aires 2002 [en línea]. [acceso 2020 Nov 03]. Disponible en: http://federicotobar.com.ar.

13. Chiara M, Ariovich A, Moro J, Di Virgilio M, Tobar F, Catenazzi A. Gestión territorial integrada para el sector salud. Los Polvorines: Universidad Nacional de General Sarmiento; 2015.

14. Medina A, Narodovski P. Estado, Integración y Salud. La gestión en red de un hospital público. Colección $\mathrm{Bi}$ tácora Argentina; 2015

15. Bossio Jc, Arias SJ, Fernández Hr. Tuberculosis en Argentina: desigualdad social y de género. Salud Colectiva 2012; 8(Supl. 1):S77-S91.

16. Spinelli H, Macías G, Darraidou V. Procesos macroeconómicos y homicidios. Un estudio ecológico en los partidos del Gran Buenos Aires (Argentina) entre los años 1989 y 2006. Salud Colectiva 2008; 4(3):283-299.

17. García GG, Tobar F. Más salud por el mismo dinero. Buenos Aires: Editorial Nuevo hacer; 1997.

18. García GG, Tobar F. Salud para los argentinos. Buenos Aires: Ediciones ISALUD; 2004.

Artículo presentado en 20/11/2020

Aprobado en 24/05/2021

Versión final presentada en 26/05/2021

Editores jefes: Romeu Gomes, Antônio Augusto Moura da Silva 
